\title{
Methotrexate and leflunomide survival in patients with psoriatic arthritis
}

Abstract: The objectives of this study were to estimate the survival rate of the most frequently used DMARDs in a cohort of PsA patients and factors associated with a higher survival rate.

Methods: Patients $\geq 18$ years of age with PsA according to CASPAR criteria, belonging to the RAPSODIA cohort were studied. Socio-demographic, clinical and treatment data were collected. Statistical analysis: Kaplan Meier survival curves and log rank were used to analyse and compare drugs' survival rate. Cox proportional analysis was performed to determine associated factors with drug survival.

Results: 87 patients with PsA were included with a median age of 52 years (IQR 40.2-61.7) and a slight female predominance $(52.9 \%)$. Seventy patients $(80.5 \%)$ received MTX, $23(32.9 \%)$ had to discontinue it due to adverse events (65\%) or treatment failure (35\%). The median survival time of MTX treatment was 13 years (IQR 8.5-17.4). The cumulative survival rate after 10 years of treatment was $55 \%$, being significantly higher among patients receiving concomitant steroid therapy (median $16.4 \pm 2.3$ years vs. $10 \pm 2$ years, $p=0.01$ ).

Of the 16 patients receiving LFN, $56.25 \%$ had to discontinue, estimating a median survival time of 6 years (IQR 1.6-10.3). The cumulative survival rate after 10 years was $35 \%$. Using a cut-off value of 50 years, elderly patients had a higher drug survival (median $5.5 \pm 1.5$ years vs. $3 \pm 1$ years, $p=0.03$ ).

Conclusion: MTX was the most frequently DMARD used. MTX cumulative survival was greater than that of LFN and was favoured by concomitant steroid therapy. LFN survival was higher amongst elderly patients.

Keywords: psoriatic arthritis $\bullet$ methotrexatehand ultrasound $\bullet$ leflunomide $\bullet$ drug survival

\section{Introduction}

Psoriatic Arthritis (PsA) is a heterogeneous disease and treatment should be tailored according to its severity. The goal of treatment for all PsA patients should be remission or low disease activity. Disease modifying anti-rheumatic drugs (DMARDs) has the potential for controlling disease activity, however, unlike their effect in other rheumatic diseases, in PsA they have not proven to be useful in preserving cartilage integrity or reduce radiographic progression [1]. Nonetheless, they are still considered first line therapy, especially for patients with polyarticular involvement or widespread skin disease [2].

Methotrexate (MTX) is the DMARD of first choice, although long term treatment is in occasions limited due to adverse events. In Rheumatoid Arthritis (RA) it has been observed that approximately $35 \%$ of the patients discontinue MTX [3], yet in PsA limited data exists regarding MTX survival rate. Leflunomide (LFN) is another DMARD increasingly used, but there is even less evidence regarding survival data and tolerance. Awareness of drug survival and reasons of treatment withdraw of such medications could maximize treatment benefit for PsA patients.

The objectives of this study were to estimate the survival rate of the most frequently DMARDs used in a cohort of PsA patients, as well as the main causes of drug discontinuation and factors associated with a higher survival rate.

\section{Patients and methods}

Patients $\geq 18$ years of age with PsA according to CASPAR criteria, belonging to the RAPSODIA cohort ('Registro de Artritis Psoriásica del IREP

\author{
Landi M*, Zaffarana CA, Cerda \\ O, Gallino Yanzi J, Schneeberger \\ EE, Carrillo I, González Guzmán \\ MC \& Citera G \\ Rheumatology Section, Instituto de \\ Rehabilitación Psicofísica, Buenos Aires, \\ Argentina
}

*Author for correspondence: gustavocitera@gmail.com 
Argentina') were studied. Socio-demographic and clinical forms of the disease were recorded. Peripheral involvement was assessed by $66 / 68$ swollen and tender joint count, and by the composite indexes DAS28 (Disease Activity Score) [4] and DAPSA (Disease Activity for Psoriatic Arthritis) [5]. Quality of life was evaluated using the questionnaires DLQI (Dermatology Life Quality Index) [6] and PsAQoL (Psoriatic Arthritis Quality of Life) [7], functional status by HAQ-A (Health Assessment Questionnaire- Argentine version) [8] and axial involvement using BASDAI (Bath Ankylosing Spondylitis Disease Activity Index) [9] and BASFI (Bath Ankylosing Spondylitis Functional Index) [10]. Furthermore, the composite index CPDAI (Composite Psoriatic Disease Activity Index) [11] was calculated. Psoriasis was studied by means of PASI score (Psoriatic Area and Severity Index) [12], and nail involvement using PNSS (Psoriatic Nail Severity Score) [13]. Physical examination also included assessment of dactilitis (measured by the difference in the circumference between two fingers or toes) and enthesitis through MASES (Maastricht Ankylosing Spondylitis Enthesitis Score) [14]. Data regarding treatment was gathered by a direct interview with the patient and from medical records in order to reduce forgetfulness bias.

Statistical analysis: Continuous variables are expressed as medians and interquartile range (IQR), while categorical data as frequencies and percentages. Mann Whitney or T test with Levene's test for homogeneity of variance were used to compare continuous variables, and Chi2 or Fisher's exact test for categorical data. Kaplan Meier survival curves and log rank were used to analyse and compare drugs' survival rate. Cox proportional analysis was performed to determine associated factors with drug survival. A p value less than 0.05 was considered significant.

\section{Results}

Of the 120 patients belonging to the RAPSODIA cohort, 87 had complete data to be included in the present analysis. Median age was 52 years (IQR 40.2-61.7) and 52.9\% were females. Median PsA duration was 10 years (IQR 6-17). The most prevalent clinical form was polyarticular (60\%), followed by decreasing order of frequency by oligo/monoarticular (32\%), spondylitic (7\%) and mutilans (1\%). Clinical characteristics of the population are depicted in Table 1.

Table 1. Sociodemographic and Clinical Characteristics

\begin{tabular}{|c|c|c|}
\hline \multicolumn{2}{|c|}{ Variable } & $n=87$ \\
\hline \multicolumn{2}{|c|}{ Female sex n (\%) } & $46(52.9)$ \\
\hline \multicolumn{2}{|c|}{ Age in years m (IQR) } & $52(40.2-61.7)$ \\
\hline \multicolumn{2}{|c|}{ PsA duration in years $m$ (IQR) } & $10(6-17)$ \\
\hline \multicolumn{2}{|c|}{ Psoriasis duration in years $\mathrm{m}$ (IQR) } & $21(14-32)$ \\
\hline \multicolumn{2}{|c|}{ DAS 28 m (IQR) } & $3.52(2.47-4.37)$ \\
\hline \multicolumn{2}{|c|}{ DAPSA m (IQR) } & $15.35(7.58-23)$ \\
\hline \multicolumn{2}{|c|}{ PASI m (IQR) } & $1.1(0.3-3.98)$ \\
\hline \multicolumn{2}{|c|}{ HAQ-A m (IQR) } & $0.87(0.25-1.25)$ \\
\hline \multicolumn{2}{|c|}{ BASDAI m (IQR) } & $4.1(1.9-7)$ \\
\hline \multicolumn{2}{|c|}{ PsAQoL m (IQR) } & $8(2-13)$ \\
\hline \multirow{4}{*}{ Clinical form of PsA } & Polyarticular n(\%) & $50(60)$ \\
\hline & Oligoarticular n(\%) & $26(32)$ \\
\hline & Spondylitic n(\%) & $6(7)$ \\
\hline & Mutilans n(\%) & $1(1)$ \\
\hline \multirow{3}{*}{ Treatment } & Methotrexate $\mathrm{n}(\%)$ & $70(80.5)$ \\
\hline & Leflunomide n(\%) & $16(18.4)$ \\
\hline & Combined n(\%) & $5(5.8)$ \\
\hline \multirow{2}{*}{ Drug survival in years } & Methotrexate m(IQR) & $13(8.57-17.42)$ \\
\hline & Leflunomide m(IQR) & $6(1.66-10.33)$ \\
\hline
\end{tabular}


Seventy patients $(80.5 \%)$ received MTX, and $23(32.9 \%)$ had to discontinue it due to adverse events $(65 \%)$ or treatment failure (35\%). The most frequent adverse events were gastrointestinal (GI) intolerance (47\%), elevated transaminase (40\%), elevated alkaline phosphatase $(7 \%)$ and nodules (7\%). The median survival time of MTX was 13 years (IQR 8.5-17.4). The cumulative survival rate after 10 years of treatment was $55 \%$, being significantly higher among patients receiving concomitant steroid therapy (median $16.4 \pm$ 2.3 years $v$ s. $10 \pm 2$ years, $\mathrm{p}=0.01$ ) Figures 1 and 2. This association was confirmed in the Cox proportional analysis.
Of the 16 patients receiving LFN, 56.25\% had to discontinue it, estimating a median survival time of 6 years (IQR 1.6-10.3). The main reasons for discontinuation were adverse events $(44.5 \%)$ and treatment failure (33.3\%). The most frequent adverse events reported were haematological disorders $(25 \%)$, elevated transaminase $(25 \%)$ and GI intolerance $(25 \%)$. The cumulative survival rate after 10 years was $35 \%$ (Figure 3). Patients age had a mayor impact in LFN survival; using a cut-off value of 50 years, elderly patients had a higher drug survival (median $5.5 \pm 1.5$ years vs. $3.3 \pm 1$ years, $\mathrm{p}=0.03)$ Figure 4 .

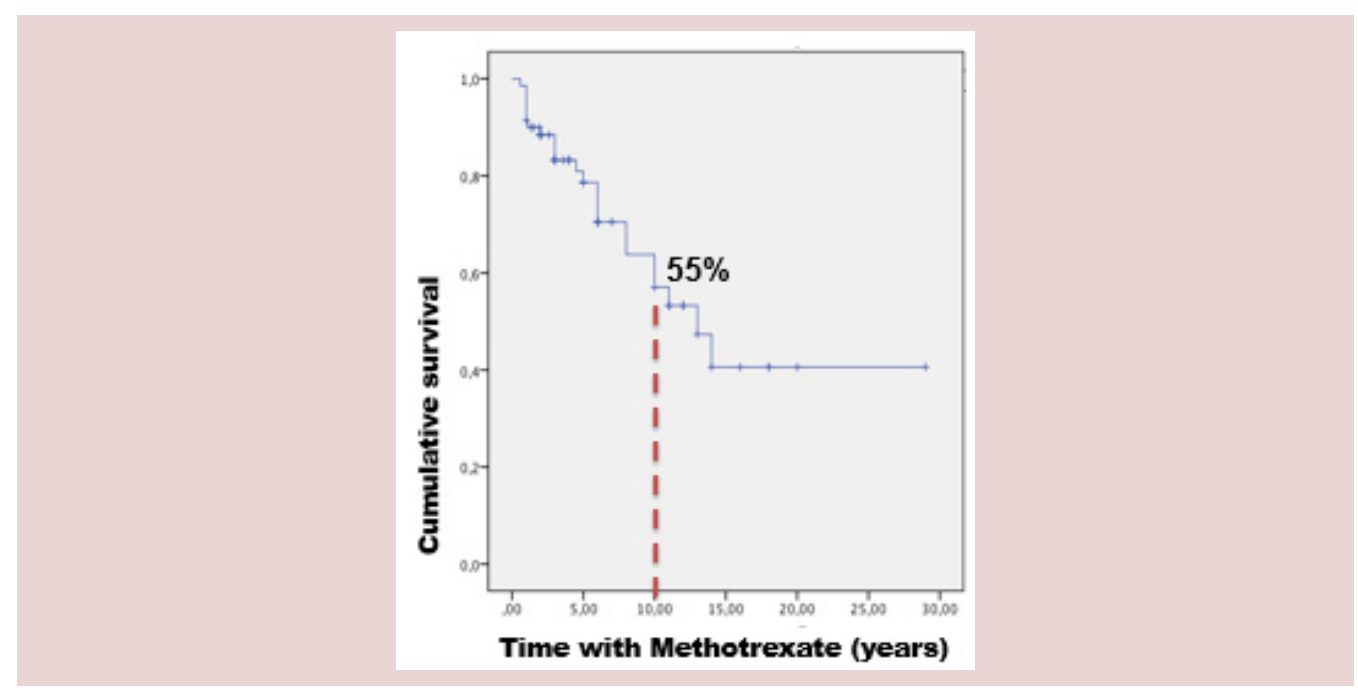

$\mathrm{m}$, median; IQR, interquartile range

Figure 1. Methotrexate Treatment Cumulative Survival

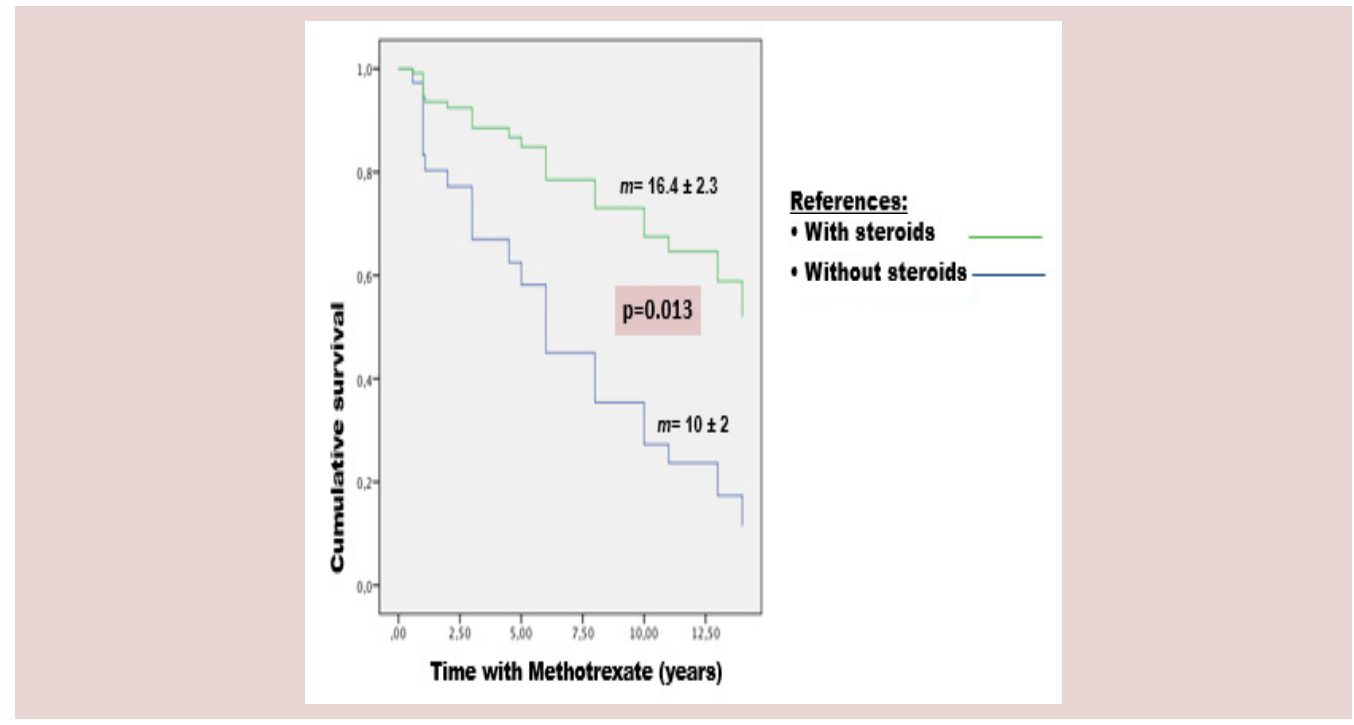

m, median

Figure 2. Cumulative Survival Rate of Methotrexate Treatment According to Concomitant Steroid Therapy 


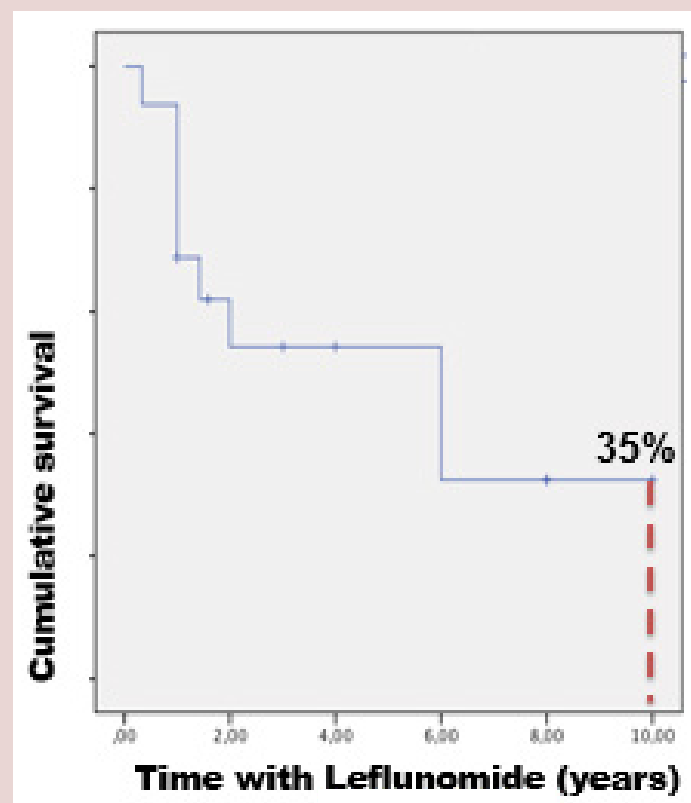

m, median; IQR, interquartile range

Figure 3. Cumulative Survival Rate of Leflunomide Treatment

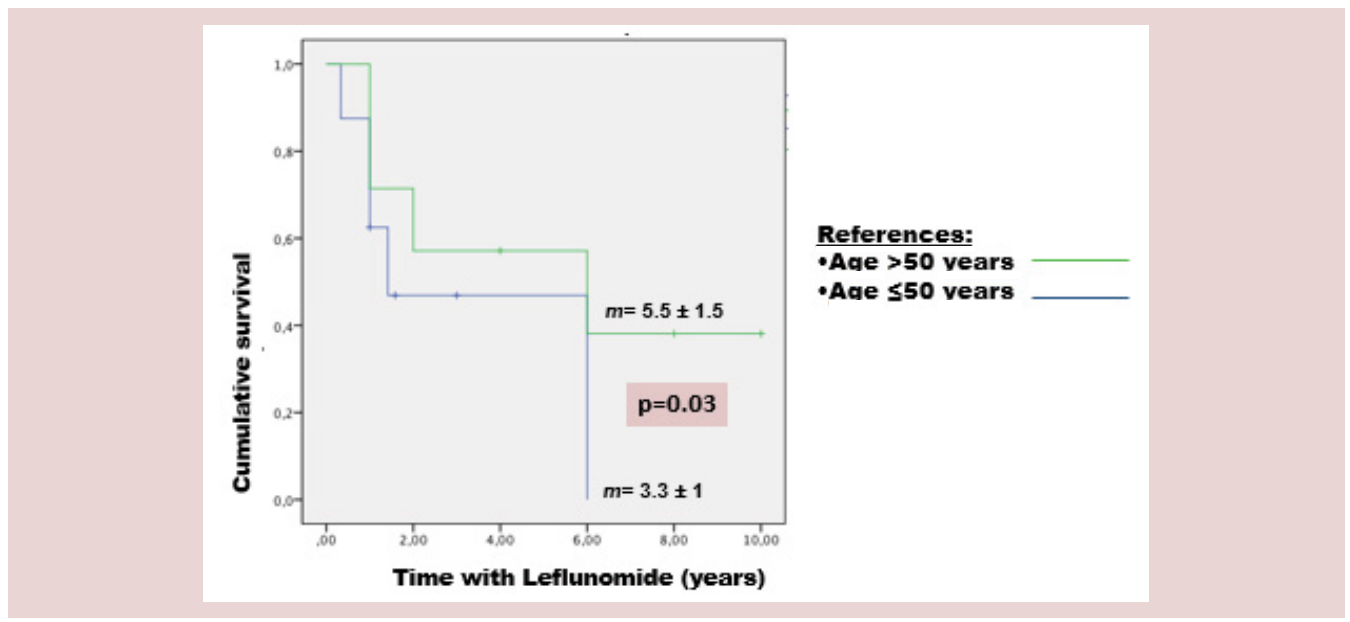

m, median

Figure 4. Cumulative Survival Rate of Leflunomide Treatment According to Patients' Age

\section{Discussion}

In this cohort of PsA patients, MTX was the most frequently DMARD used (80.5\%), followed by LFN (18.5\%). The cumulative survival rate of MTX after 10 years of treatment was $55 \%$, being significantly higher among patients receiving concomitant steroid therapy. In contrast, one third of the patients receiving LFN continued treatment after 10 years, with a higher survival rate amongst patient's $\geq 50$ years of age. Adverse events and loss of efficacy were the main reasons of discontinuation for both drugs.

The data provided by our study show a high MTX survival rate in PsA patients, with a median of 13 years. An Italian PsA cohort observed that $60 \%$ of the patients continue MTX after 3 years of treatment [15]. Lie et al., described a similar MTX survival rate in PsA as in RA [16]. Data from United Kingdom (UK) estimated a mean MTX treatment duration of 18.6 months [3], whilst Dalkilic et al, studying mainly patients with RA and few with PsA, calculated and average time to MTX discontinuation of only 8.1 months [17].

The main reason of MTX discontinuation in our cohort was due to adverse events, being GI intolerance the most frequently reported event. These data are similar to those observed in UK 
[3]. Conversely, Dalkilic et al described a lower GI intolerance to MTX, corresponding to $28.6 \%$ [17]. The Italian cohort only reported $10 \%$ of adverse events and patients had a good tolerance to MTX [15]. Comparisons between these cohort is difficult due, among other reasons, to loss of follow up, the variable number of patients and the median MTX dose used included in each study.

Unlike to Lie et al., that found that a longer disease duration, younger age, worse functional status and worse disease assessment by the patients were independent predictors of MTX withdraw, our study only observed a longer MTX survival in those patients taking concomitant steroid therapy [16]. Due to the study design, it is hard to find a definite answer for this association. A possible explanation to this finding could be that combined steroid treatment facilitates comprehensive disease control and consequently fewer patients abandon medication due to lack of efficacy. We have checked this difference in the 23 patients who discontinued due to adverse events and found no dissimilarities. However, the number is very small and the possibility of a type II error is quite high.

LFN has demonstrated to be useful in controlling joint and skin manifestations in PsA [18]. It has been recently reported that approximately $50 \%$ of patients treated with LFN show disease improvements after one year of treatment [19]. A prospective study including PsA patients from daily practice described that LFN improves peripheral, skin and nail involvement as well as fatigue. The rate of discontinuation after 24 weeks of treatment was $12.3 \%$, and adverse events were reported in $12.1 \%$ of the patients [20]. In addition, a retrospective study from Malesci et al estimated a discontinuation rate of $29 \%$ after 24 months of treatment with LFN [21]. GI intolerance should been analyzed in the light of previous DMARD use. In our cohort, $65 \%$ of the patients had previously been treated with MTX and to a lesser extend with 2 DMARDs. The striking observation that elderly patients had a higher LFN survival rate cannot be explained. Knowing the safety profile of this drug; the increased risk of high blood pressure, alopecia, and GI intolerance among other common adverse events, one would tend to think that older patents would present less tolerance.

Limitations to our study include its retrospective nature, the study design that does not allow a direct comparison between the survival rates of both drugs, and the fact that GI intolerance was defined according to the treating physician criteria.

Despite the fact that the beneficial influence of DMARDs in radiographic progression has not yet been proven, they are able to control disease activity in a considerable number of patients, motive of their consideration as first-line drugs for PsA [22]. Nevertheless, the lack of response or the incidence adverse events should not delay therapeutic decisions to other drugs with different modes of actions.

To the best of our understanding, this is the first study to assess the survival rate of DMARDs in PsA patients from daily practice in Argentina. MTX was the most frequently DMARD used followed by LFN. MTX cumulative survival was greater than that of LFN and was favoured by concomitant steroid therapy, while LFN survival was higher amongst elderly patients.

\section{References}

1. Abu-Shakra M, Gladman DD, Thorne JC et al. Longterm methotrexate therapy in psoriatic arthritis. J. Rheumatol. 22(2), 241-245 (1995).

2. Finlay AY, Khan GK. Dermatology Life Quality Index (DLQI)-a simple practical measure for routine clinical use. Clin. Exp. Dermatol. 19(3), 210-6 (1994).

3. Nikiphorou E, Negoescu A, Fitzpatrick JD et al. Indispensable or intolerable? Methotrexate in patients with rheumatoid and psoriatic arthritis: a retrospective review of discontinuation rates from a large UK cohort. Clin. Rheumatol. 33(5), 609-14 (2014).

4. Felson DT, Anderson JJ, Boers M et al. The American College of Rheumatology preliminary core set of disease activity measures for rheumatoid arthritis clinical trials. The Committee on Outcome Measures in Rheumatoid Arthritis Clinical Trials. Arthritis. Rheum. 36(6), 729-40 (1993).

5. Schoels M, Aletaha D, Funovits J et al. Application of the DAREA/DAPSA score assessment of disease activity in psoriatic arthritis. Ann. Rheum. Dis. 69(8), 1441-7 (2010).

6. Finlay AY, Khan GK. Dermatology Life Quality Index (DLQI): a simple practical measure for routine clinical use. Clin. Exp. Dermatol. 19(3), 210-6 (1994).

7. McKenna SP, Doward LC, Whalley D et al. Development of the PsAQoL: a quality of life instrument specific to psoriatic arthritis. Ann. Rheum. Dis. 63(2), 162-9 (2004).

8. Citera G, Arriola MS, Maldonado-Cocco JA et al. Validation and crosscultural adaptation of an Argentine Spanish version of the health assessment questionnaire disability index. J. Clin. Rheumatol. 10(3), 110-5 (2004).

9. Garret S, Jenkinson T, Kennedy LG et al. A new approach to defining disease status in ankylosing spondylitis: the Bath Ankylosing Spondylitis Disease Activity Index. J. Rheumatol. 21(12), 2286-91 (1994). 
10. Calin A, Garrett S, Whitelock H et al. A new approach to defining functional ability in ankylosing spondylitis: the development of the Bath Ankylosing Functional Index. J. Rheumatol. 21(12), 2281-5 (1994).

11. Mumtaz A, Gallagher P, Kirby B et al. D. J. Rheumatol evelopment of a preliminary composite disease activity index in psoriatic arthritis. Ann. Rheum. Dis. 70(2), 272-7 (2011)

12. Marks R, Barton SP, Shuttleworth D et al. Assessment of disease progression in psoriasis. Arch. Dermatol. 125(2), 235-40 (1989).

13. Williamson L, Dalbeth N, Dockerty JL et al. Extended report: nail disease in psoriatic arthritis--clinically important, potentially treatable and often overlooked. Rheumatology. 43(6), 790-4 (2004).

14. Heuft-Dorenbosch L, Spoorenberg A, van Tubergen A et al. Assessment of enthesitis in ankylosing spondylitis. Ann. Rheum. Dis. 62(2), 127-32 (2003).

15. Ricci M, De Marco G, Desiati F et al. Long-term survival of methotrexate in psoriatic arthritis. Rheumatismo. 61(2), 125-31 (2009).

16. Lie E, van der Heijde D, Uhlig $\mathrm{T}$ et al. Effectiveness and retention rates of methotrexate in psoriatic arthritis in comparison with methotrexate-treated patients with rheumatoid arthritis. Ann. Rheum. Dis. 69(4), 671-6 (2010).

17. Dalkilic E, Sahbazlar M, Gullulu M et al. The time course of gastric methotrexate intolerance in patients with rheumatoid arthritis and psoriatic arthritis. Mod. Rheumatol. 23(3), 525-8 (2013).

18. Kaltwasser JP, Nash P, Gladman D et al. Efficacy and safety of leflunomide in the treatment of psoriatic arthritis and psoriasis: a multinational, double-blind, randomized, placebo-controlled clinical trial. Arthritis. Rheum. 50(6), 1939-50 (2004)

19. Asiri A, Thavaneswaran A, Kalman-Lamb G et al. The effectiveness of leflunomide in psoriatic arthritis. Clin. Exp. Rheumatol. 32(5), 728-31 (2014).

20. Behrens F, Finkenwirth C, Pavelka K et al. Leflunomide in psoriatic arthritis: results from a large European prospective observational study. Arthritis. Care. Res. 65(3), 464-70 (2013).

21. Malesci D, Tirri R, Buono Ret al. Leflunomide in psoriatic arthritis: a restrospective study of discontinuation rate in daily clinical practice compared with methotrexate. Clin. Exp. Rheumatol. 25(6), 881-4 (2007).

22. Soriano ER. The actual role of therapy with traditional disease-modifying antirheumatic drugs in psoriatic arthritis. J. Rheumatol. 89, 67-70 (2012). 